\title{
DIVERSIDAD DE FLEBOTOMINOS EN UNA ZONA ENDÉMICA DE LEISHMANIASIS VISCERAL AMERICANA EN VENEZUELA
}

\author{
Luis Eduardo Traviezo Valles ${ }^{1, a}$
}

\section{RESUMEN}

\begin{abstract}
Con el objetivo de estudiar la ecología de la flebotomofauna en la población de La Rinconada, perteneciente al estado de Lara, Venezuela, zona endémica donde se han descrito casos de leshmaniasis visceral americana (LVA), se realizó un estudio longitudinal durante cinco meses, en los que una noche por mes se capturó flebótomos en los alrededores de una casa en la que previamente se había informado de un caso de LVA. Se encontró una diversidad de seis especies, de las cuales solo dos eran antropofílicas, siendo Lutzomyia pseudolongipalpis la más abundante (90\%). El pico de abundancia se observó en julio, mes seco que precede a los meses más lluviosos del año. Aunque en nuestra muestra no encontramos evidencia de que los flebótomos estuvieran infectados por Leishmania; la evidencia presentada es importante para el mejor entendimiento de un vector que participa de la transmisión de la LVA.
\end{abstract}

Palabras clave: Leishmaniasis visceral; Lutzomyia; Enfermedades endémicas; Dípteros (fuente: DeCS BIREME).

\section{DIVERSITY OF PHLEBOTOMINAE IN AN ENDEMIC ZONE OF AMERICAN VISCERAL LEISHMANIASIS IN VENEZUELA}

\begin{abstract}
In order to study the ecology of phlebotominae in the population of La Rinconada, in the state of Lara, Venezuela, endemic zone of Leishmaniasis, where cases of American Visceral Leshmaniasis (AVL) have been described, a longitudinal study was conducted during five months, and the presence of phlebotominae was identified one night per month in the surrounding areas of a home where an AVL case had been reported. Six species were found, of which only two were anthropophilic. The most abundant species was Lutzomyia pseudolongipalpis (90\%), the abundance peak was observed in July - a dry month preceding the rainiest months of the year. Although in our sample we have not found any evidence that phlebotominae were infected by Leishmania; evidence presented is important for a better understanding of a vector that participates in AVL transmission.
\end{abstract}

Key words: Leishmaniasis, visceral; Psychodidae; Endemic diseases; Diptera (source: MesH NLM).

\section{INTRODUCCIÓN}

Los flebótomos son una subfamilia de dípteros nematóceros de la familia Psychodidae, conocidos como el único vector en la transmisión de la leishmaniasis a través de la picadura de los flebótomos hembras ${ }^{(1)}$. De ellos se han descrito más de 800 especies distribuidas a nivel mundial, con preferencias por climas cálidos y húmedos, por lo que aumentan característicamente en época de lluvias ${ }^{(1)}$.
En América la leishmaniasis visceral americana (LVA) es causada por Leishmania infantum y transmitida al hospedador vertebrado principalmente por la hembra del flebótomo Lutzomyia longipalpis ( $L$. longipalpis); se ha descrito una incidencia de 16000 casos anuales (2) y se ha señalado que existirían, al menos, 1,5 millones de personas en riesgo de infección, las cuales se distribuyen fundamentalmente en Brasil, Venezuela y Argentina; con discretos focos

\footnotetext{
Univesidad Centrooccidental "Lisandro Alvarado". Lara, Venezuela.

a Licenciado en Bioanálisis magister en Protozoología.

Recibido: 15-03-12 Aprobado: 14-11-12
} 
en Paraguay, Bolivia, Perú, Colombia, El Salvador, Honduras, Guatemala y México, siendo las áreas rurales las más afectadas ${ }^{(3-7)}$.

El primer caso de LVA en Venezuela fue descrito en 1941 por Martínez y Pons ${ }^{(3)}$, seguido por los trabajos de Pifano ${ }^{(6)}$ y J.W. Torrealba ${ }^{(8)}$ quienes estudiaron los tres eslabones de la cadena de trasmisión ( $L u$. longipalpis como vector, los canes como reservorios domésticos y la población humana como la expuesta al riesgo). En este país la enfermedad se distribuye geográficamente en tres focos (central, oriental y occidental); es de interés para nuestra investigación el foco occidental en donde se ubica el estado de Lara, además de Falcón, Portuguesa, Zulia y Trujillo ${ }^{(3-7)}$.

El estado de Lara se encuentra ubicado en la región centro occidental, a una altitud promedio de 570 metros, la temperatura media anual fluctúa entre 19 y $29{ }^{\circ} \mathrm{C}$, en él predominan dos tipos de clima: el tropical y el árido templado. La sequedad del ambiente es típica, ya que la evaporación supera a las precipitaciones, estas alcanzan $650 \mathrm{~mm}$ de promedio anual, con lluvias que caen en épocas diferentes de acuerdo con el lugar. Entre los años 1995 a 1999 el estado Lara presentó la mayor mortalidad registrada en Venezuela por LVA (siete decesos). Además, entre el 2000 al 2005, Lara se encontró entre los tres primeros estados con mayor incidencia de LVA, pues se informó 53 casos distribuidos en los municipios Torres, Jiménez y Urdaneta ${ }^{(7-10)}$.

En el año 2004, en la población rural de "La Rinconada" perteneciente al municipio Torres se presentaron tres casos de LVA, durante el estudio de estos casos se describió un nuevo vector en la transmisión de la LVA, la Lutzomyia pseudolongipalpis (Lu. Pseudolongipalpis) una especie del Grupo Verrucarum (10). Este hecho convirtió a esta comunidad en un ecotopo de singular importancia en el estudio de los vectores transmisores de la LVA y, por ende, de la ecología de estos vectores; el estudio de este ecotopo permitirá comprender mejor la dinámica de la transmisión de la Leishmania infantum y así prevenir su diseminación (7-9). La Rinconada se ubica en los $10^{\circ} 00^{\prime} 15^{\prime \prime} \mathrm{N}$ y $69^{\circ} 57^{\prime} 00^{\prime \prime} \mathrm{O}$; su clasificación climática es de tipo árido templado, con una zona de vida del tipo bosque seco premontano, caracterizada por una precipitación media anual de $636,6 \mathrm{~mm}$ y temperatura media anual de $25,4{ }^{\circ} \mathrm{C}$, un período seco (enero y mayo) y dos períodos de lluvia (mayo y octubre) ${ }^{(7)}$.

Consideramos que la información existente sobre $L u$. pseudolongipalpis aún es escasa dentro de la literatura biomédica, sobre todo en relación a los hábitos y distribución horaria de los flebótomos, en particular de la Lu. pseudolongipalpis; por ello el presente estudio tiene como objetivo de conocer la diversidad de flebótomos, su distribución dentro y fuera de la casa, la distribución de especies y otros elementos que favorecen su presencia.

\section{EL ESTUDIO}

Se realizó un estudio de tipo longitudinal en la población rural de La Rinconada durante cinco meses, comprendidos entre febrero de 2005 y abril de 2006. Para la determinación de los límites de las áreas de interés en nuestro estudio, se tomó como punto de referencia una casa en la cual se había informado previamente un caso de LVA, en función a ella se delimitaron tres áreas de estudio: intradomiciliaria, peridoméstica (diez metros alrededor de la vivienda) y selvática (cincuenta metros alrededor de la vivienda).

Para la captura de los flebótomos se emplearon seis trampas de luz Communicable Disease Center (CDC), se distribuyó dos trampas en cada área de interés en estudio, las que permanecían encendidas durante doce horas (desde las $19.00 \mathrm{~h}$ hasta las $07.00 \mathrm{~h}$ del día siguiente); a la primera de ellas se le cambió la malla una vez por noche, en tanto que a la segunda, cada hora. Esto permitió detectar la hora de mayor actividad y relacionarla con sus preferencias domésticas, peridomésticas o selváticas. En total se hicieron cinco capturas, cada una en meses distintos, una sola noche por mes. Se determinó que las capturas se realizasen en noches de luna nueva o próxima a esta fase lunar, para que la luz de las CDC tuviera su máxima atracción; además, se procuró que las noches de captura la vivienda permaneciera con las luces apagadas para así dejar como única fuente de luz la trampa CDC.

Los insectos obtenidos fueron ubicados en jaulas con cubiertas de organdí, dentro de cavas de poliuretano con humedad, para trasladarlos al siguiente día al laboratorio (ubicado en Barquisimeto, capital del Estado de Lara); luego fueron colocados en bolsas con cloroformo y congelación para anestesiar a los insectos capturados; posteriormente, se separaron los flebótomos del resto de artrópodos; seguidamente, fueron clasificados por procedencia e inmediatamente sumergidos en alcohol al $70 \%$ para preservarlos hasta el día del examen. En dicho día los insectos se transfirieron individualmente a una solución de $\mathrm{KOH}$ al $10 \%$, en la que permanecieron por $24 \mathrm{~h}$ para una digestión parcial de sus estructuras externas. Seguidamente, eran colocados uno a uno con aguja entomológica en láminas porta objeto, se cubrían con laminillas $22 / 22 \mathrm{~mm}$, para ser observadas 
Tabla 1. Diversidad y frecuencia de la flebotomofauna en La Rinconada, estado Lara, Venezuela.

\begin{tabular}{|c|c|c|c|c|c|c|c|c|c|c|c|c|}
\hline & \multicolumn{2}{|c|}{ Feb. 2005} & \multicolumn{2}{|c|}{ Abr. 2005} & \multicolumn{2}{|c|}{ May.05 } & \multicolumn{2}{|c|}{ Jul. 2005} & \multicolumn{2}{|c|}{ Abr. 2006} & \multicolumn{2}{|c|}{ TOTAL } \\
\hline & N. ${ }^{\circ}$ & $(\%)$ & N..$^{\circ}$ & (\%) & N. ${ }^{\circ}$ & (\%) & $\mathrm{N} .^{\circ}$ & (\%) & N..$^{\circ}$ & (\%) & $\mathbf{N} .^{\circ}$ & $(\%)$ \\
\hline \multicolumn{13}{|c|}{ Lu. pseudolongipalpis } \\
\hline Hembra & 4 & $(11,4)$ & 82 & $(54,7)$ & 92 & $(48,9)$ & 45 & $(20,8)$ & 144 & $(54,9)$ & 367 & $(43,1)$ \\
\hline Macho & 6 & $(17,1)$ & 32 & $(21,3)$ & 91 & $(48,4)$ & 158 & $(73,2)$ & 114 & $(43,5)$ & 401 & $(47,1)$ \\
\hline Lu. evansi & 2 & $(5,7)$ & & - & 1 & $(0,5)$ & 13 & $(6,0)$ & & - & 16 & $(1,9)$ \\
\hline Lu. trinidadensis & 10 & $(28,6)$ & 33 & $(22,0)$ & & - & & - & 2 & $(0,8)$ & 45 & $(5,3)$ \\
\hline Lu. cayennensis & 13 & $(37,1)$ & 3 & $(2,0)$ & 1 & $(0,5)$ & & - & 2 & $(0,8)$ & 19 & $(2,2)$ \\
\hline Lu. venezuelensis & & - & & - & 2 & $(1,1)$ & & - & & - & 2 & $(0,2)$ \\
\hline Lu. marajoensis & & - & & - & 1 & $(0,5)$ & & - & & - & 1 & $(0,1)$ \\
\hline TOTAL & 35 & (100) & 150 & $(100)$ & 188 & $(100)$ & 216 & (100) & 262 & $(100)$ & 851 & (100) \\
\hline
\end{tabular}

al microscopio óptico con aumento de 100 y 400X para ubicar elementos específicos como las espermatecas y la armadura del cibario (hembras), con lo cual se determinó la especie ${ }^{(10)}$. También se observaron con objetivo de 10X y posteriormente con 40X, los tractos digestivos de todas las hembras disecadas, con el propósito de detectar infección natural con Leishmania sp. Además, se recolectó información sobre las precipitaciones mensuales informadas por la Estación Curarigua del Ministerio del Ambiente de Venezuela a $627 \mathrm{~m}$ de altura ${ }^{(6)}$.

\section{HALLAZGOS}

Se atraparon un total 851 flebótomos en cinco noches de captura; se identificó seis especies, siendo Lutzomyia pseudolongipalpis la especie más abundante (90\%) a diferencia de Lutzomyia evansi (Núñez-Tovar, 1924), que solo presentó un $1,9 \%$ del total de capturas, el resto de las especies colectadas no son antropofílicas (Tabla 1).

Con respecto a la abundancia relacionada al sexo de Lu. pseudolongipalpis se apreció que los machos eran más abundantes que las hembras (relación de 1,1:1), siendo esta diferencia más clara en la captura de julio (relación de 3,5:1). Sin embargo, durante el mes de abril, antes de las lluvias, las hembras invirtieron esta proporción (relación: 1:1,3).

Con respecto a la distribución de la flebotomofauna, de acuerdo con los ambientes: doméstico, peridoméstico y selvático, fue de $24,5 \%$ (126); 17,7\% (88), y 56,9\% (282) respectivamente, donde se nota que el mayor porcentaje era en la zona montañosa de abundante vegetación de tipo bosque seco tropical, seguido por el interior de la casa. Es importante señalar la ausencia de especies no antropofílicas dentro de la casa, estas se consiguieron solo en el peridomicilio y la zona selvática.
Con relación a la distribución de las capturas según la zona y hora y la actividad horaria, se encontró que la mayor abundancia de flebótomos se da hasta antes de la media noche, para posteriormente disminuir progresivamente durante la madrugada. Se encontró dos picos de abundancia, el primero de 21.00 a 22.00 h y el segundo de 23.00 a 24.00 h. En la evaluación por áreas de interés se observó que dentro del área intradomiciliaria la mayor abundancia fue de 11.00 a $12.00 \mathrm{~h}$, en tanto que en el área peridomiciliaria la mayor abundancia fue de 24.00 a $01.00 \mathrm{~h}$, y en el área selvática de 21.00 a $22.00 \mathrm{~h}$.

En cuanto a Lu. Pseudolongipalpis se observa que las hembras presentan dos picos, el primero de 21.00 a $22.00 \mathrm{~h}$ y el segundo de 23.00 a $24.00 \mathrm{~h}$; mientras que los machos mantuvieron su abundancia de 19.00 a 22.00 h y de 23.00 a 01.00 h. Lu. evansi solo se presentó de 21.00 a $23.00 \mathrm{~h}$.

La relación de la abundancia de las dos especies antropofílicas con la precipitación registrada para las mismas fechas en la Estación Curarigua ${ }^{(5)}$, se observó cómo la presencia de hembras de $L u$. pseudolongipalpis se acrecienta con el aumento de la precipitación mensual del mes de captura, detectándose cómo en abril de 2006 el pico fue mayor al mismo mes del año anterior, a pesar de presentar una precipitación menor que en abril de 2005. Los machos presentaron mayor abundancia en el mes menos húmedo. También se nota que $L u$. evansi solo aparece en los meses más secos o de menor precipitación y mayor temperatura.

Con respecto a la observación de los tractos digestivos de las hembras disecadas (todas las especies capturadas), para detectar infección natural, no se consiguió ninguna infectada con Leishmania sp. 


\section{DISCUSIÓN}

En La Rinconada el primer elemento que resalta es la mayor abundancia de flebótomos capturados y la diversidad de estos (seis especies), con respecto al análisis en otras zonas endémicas de LVA en Venezuela (10,11), en solo cinco noches de captura, aunque en periodos diferenciados. Resalta aun más que esta abundancia se da a expensas de Lu. pseudolongipalpis, hecho que es preocupante dado que esta especie ha sido incriminada como transmisora de LVA. Sin embargo, en nuestro estudio se ha detectado una menor proporción de esta especie en relación a lo descrito en otros trabajos, tales como en Quebrada Grande en el mismo estado Lara $(91 \%){ }^{(6)}$ y en trabajos anteriores en la misma Rinconada $(>95 \%){ }^{(11,12)}$. La segunda especie más abundante fue $L u$. evansi señalada también como transmisora de LVA, cuya presencia es menor a la informada en el municipio Urdaneta del estado Lara $(51,1 \%)$ y en Valencia del estado Carabobo $(72,9 \%)^{(9,13)}$, pero mayor a lo señalado en el estado Anzoátegui $(0,5 \%)^{(6)}$.

Con respecto a las otras especies identificadas, se tiene que $L u$. trinidadensis y Lu. cayennensis, a pesar de no ser de hábitos antropofílicos, ha sido encontrada naturalmente infectada con tripanosomatídios en Lara (Venezuela) y Colombia, lo cual las podrían incriminar, bajo ciertas condiciones, en la transmisión ${ }^{(13,14)}$.

Con respecto al mejor lugar de captura, la zona selvática presentó mayor abundancia, seguida por la doméstica; sin embargo, cabe resaltar que en esta última solo se detectó presencia de especies antropofílicas, lo cual indica que hay mayor riesgo cuando las personas penetran en zonas distantes de las casas y próximas a los bosques secos tropicales, hecho que es similar a estudios previos que señalan igualmente a las zonas selváticas como de mayor riesgo de picadura por alguno de estos flebótomos ${ }^{(15,16)}$; pero que difiere a lo informado en zonas endémicas de leishmaniasis, tanto visceral como cutánea, donde se ha señalado a la zona peridoméstica como la de mayor riesgo ${ }^{(8,15)}$.

La actividad horaria tuvo dos picos máximos antes de las $24.00 \mathrm{~h}$, para ir decreciendo paulatinamente después de la media noche. Otros autores describen un comportamiento distinto de Lu. pseudolongipalpis en zonas del estado Lara, pues refieren que la actividad fue constante durante toda la noche, mientras que para Lu. longipalpis la actividad horaria en ecotopos de Margarita y del estado Guárico, es más abundante antes de las $24.00 \mathrm{~h}{ }^{(4,16)}$. Desglosando por zonas, en la selvática el pico de mayor abundancia es de 20.00 a $22.00 \mathrm{~h}$, mientras que dentro de las casas es de 23.00 a $24.00 \mathrm{~h}$, pareciera por la diferencia de los picos que, probablemente, las primeras horas de las noche las utilizan los flebótomos para aproximarse a las viviendas y posteriormente penetrar o mantenerse peridomésticamente, para luego retirarse paulatinamente.

Cuando se desglosa la actividad horaria por especie y sexo, se observa cómo las hembras de $L u$. pseudolongipalpis presentan dos picos de 19.00 a $22.00 \mathrm{~h}$ y de 23.00 a $24.00 \mathrm{~h}$, mientras que Lu. evansi aprovecha la disminución de $L u$. pseudolongipalpis de 22.00 a $23.00 \mathrm{~h}$ para obtener su máxima abundancia, al igual que $L u$. trinidadensis que aparece desde las 23.00 a $03.00 \mathrm{~h}$, aprovechando la disminución significativa de Lu. pseudolongipalpis, esto fue descrito en Costa Rica para Lu. longipalpis que aumentaba en las primeras horas de la noche para posteriormente decrecer y este nicho ecológico es aparentemente ocupado por Lu. evansi con un menor número (17); sin embargo, es de hacer notar que después de la media noche, al disminuir la temperatura los dípteros disminuyen su actividad ya que por ser poiquilotermos, la disminución de la temperatura ambiental en la madrugada los inactiva ${ }^{(7,17)}$.

Al relacionar la abundancia de sexo de $L u$. pseudolongipalpis se estima que hubo mayor cantidad de machos, mucho mayor a lo encontrado anteriormente en estudios en esta zona (9,5\%), pero menor a lo señalado normalmente para machos de Lu. longipalpis en Costa Rica $(91 \%)$ o Colombia $(81,8 \%)^{(6,17)}$, esta abundancia significativa de machos se presentó solo en una captura, la correspondiente a julio 2005 , ya que en el resto del año, siempre hubo abundancia de hembras. Este aumento se presentó al final de un período seco que precedía el comienzo del período lluvioso, lo cual se ha descrito en esta zona donde las pupas de los machos eclosionan primero al principio de las lluvias, luego de períodos secos largos ${ }^{(9,15)}$.

Al relacionar la precipitación con la abundancia, se aprecia cómo en las capturas de julio 2005 y abril 2006, estas fueron más abundantes, ambas correspondían a meses secos que preceden el comienzo de las lluvias, esto se asemeja a informes de Lu. longipalpis de Costa Rica y Brasil que refieren mejores capturas en meses secos que preceden a los húmedos ${ }^{(7,18)}$, lo contrario se señala en vectores de leishmaniasis cutánea de zonas húmedas de Venezuela y Argentina que esperan la disminución de las lluvias, períodos más secos y calientes, para lograr picos de abundancia ${ }^{(19,20)}$. 
El estudio tiene limitaciones. Aunque la selección de los lugares de captura se realizó priorizando áreas donde la probabilidad de encontrar flebótomos infectados por Leishmania, solo se procedió a la recolección una vez por mes, en meses espaciados y sin incluir el periodo de lluvias. Sin embargo, ello también permitió capturar una mayor diversidad e identificar comportamientos estacionales. De otro lado, la identificación de las especies se realizó a través de observación directa, existen actualmente métodos de identificación genética de las especies de Leishmania, lo cual habría permitido una mejor clasificación y, eventualmente, encontrar más especies; sin embargo, el equipo observador pudo identificar incluso especies de infrecuente aparición y al incluir varios periodos, la probabilidad de identificar más especies fue mayor.

En conclusión, se encontró una diversidad de seis especies, de las cuales solo dos de ellas eran antropofílicas, siendo Lutzomyia pseudolongipalpis la más abundante (90\%), el pico de abundancia se observó en julio, mes seco que precede a los meses más lluviosos del año. Aunque en esta muestra no se encontró evidencia de que los flebótomos estuvieran infectados por Leishmania; la evidencia presentada es importante para el mejor entendimiento de un vector que participa de la transmisión de la LVA, por lo que es necesario incrementar las medidas preventivas y de control de vectores tanto dentro como fuera de las casas para evitar el aumento en la incidencia de LVA.

Contribuciones de los autores: LT participó de la concepción, diseño del estudio, análisis de datos y de la redacción del artículo, así como de la aprobación de la versión final.

Fuentes de financiamiento: CDCHT VCLA proyecto 017-ME2005

Conflictos de interés: el autor declara no tener conflictos de interés.

\section{REFERENCIAS BIBLIOGRÁFICAS}

1. Cabrera O, Mosquera L, Santamaria E, Ferro C. Flebótomos (Diptera: Psychodidae) del departamento de Guaviare, Colombia, con nuevos registros para el país. Biomedica. 2009;29:73-86.

2. Alvar J, Vélez ID, Bern C, Herrero $\mathrm{M}$, Desjeux $\mathrm{P}$, Cano $\mathrm{J}$, et al. Leishmaniasis worldwide and global estimates of its incidence. PLoS One. 2012;7(5):e35671

3. Martínez N, Pons R. Primer caso de Kala-azar en Venezuela. Gac Med Caracas. 1941;48:329-32.

4. Aguilar CM, Fernández E, Fernández R, Cannova DC, Ferrer E, Cabrera Z, et al. Urban visceral leishmaniasis in Venezuela. Mem Inst Oswaldo Cruz. 1998;93(1):15-6.

5. Ulrich M, Zerpa O, Convit J. Leishmaniasis visceral humana $\mathrm{y}$ canina en Margarita. En: Memorias del Simposio de Leishmaniasis. Barquisimeto. 2002. 88-100.

6. Pífano F. Estado actual del Kala-azar en Venezuela. Archivos Venezolanos de Patología Tropical y Parasitología Médica. 1954;2:213-9.

7. Traviezo L. Flebotomofauna asociada a casos de leishmaniasis visceral en las poblaciones de La Rinconada y Quebrada Grande, Estado Lara. Barquisimeto: Decanato de Ciencias de la Salud, Universidad Centroccidental Lisandro Alvarado; 2008.

8. Torrealba J. Observaciones sobre diagnóstico terapéutica y evolución de la leishmaniasis humana y canina [Tesis doctoral]. Valencia: Decanato de Ciencias de la Salud, Universidad de Carabobo; 1970.

9. Traviezo L, Díaz A, Rodríguez R, Urdaneta R. Características Biológicas de Lutzomyia pseudolongipalpis (Diptera: Psychodidae), posible vector de leishmaniasis visceral en la población de La Rinconada, estado Lara. Bol Med Post. 2003;19(4):23540.

10. Vargas E, Añez N, Rojas A, Crisante G, Yépez J. Estudio epidemiológico de leishmaniasis visceral en El Limón, al Norte del estado Lara, Venezuela. Bol Mal y Salud Amb. 2004;44:1016.

11. Arrivillaga JC, Feliciangeli MD. Lutzomyia pseudolongipalpis: the first new species within the longipalpis (Diptera: Psychodidae: Phlebotominae) complex from
La Rinconada, Curarigua, Lara State, Venezuela. J Med Entomol. 2001;38(6):783-90.

12. Young D, Duncan M. Guide to the identification and geographic distribution of Lutzomyia sand flies in Mexico the West Indies, Central and South America (Diptera: Psychodidae). Mem Am Entomol Inst. 1994;54:1-881.

13. Agrela I, Sanchez E, Gomez B, Feliciangeli M. Feeding behavior of Lutzomyia pseudolongipalpis (Diptera: Psichodidae), a putative vector of visceral leishmaniasis in Venezuela. J Med Entomol. 2002;39(3):440-5.

14. Bonfante-G R, Urdaneta R, Urdaneta I, Rodríguez R, Perdomo R. Flebotomo fauna en dos focos endémicos de leishmaniasis visceral americana en el municipio Torres, estado Lara, Venezuela. Rev Científ FCV-LUZ. 2007;17(2):173-7.

15. Cochero S, Anaya Y, Díaz Y, Paternina M, Luna A, Paternina L, et al. Infección natural de Lutzomyia cayennensis cayennensis con parásitos tripanosomatídeos (Kinetoplastida: Trypanosomatidae) en Los Montes de María, Colombia. Rev Cubana Med 
Trop. 2007;59(1):46-50.

16. Traviezo L. Flebotomofauna al sureste del estado Lara, Venezuela. Biomedica. 2006;26(1):73-81.

17. Feliciangeli MD, Arrivillaga JC, Bravo A, Arias F. Activity of Lutzomyia pseudolongipalpis and L. longipalpis s.l. (Diptera: Psychodidae) in Venezuela. Parasite. 2004;11(3):273-8.

18. Zeledon R, Murillo J, Gutiérrez H. Observaciones sobre la ecología de Lutzomyia longipalpis (Lutz \& Neiva, 1912) y posibilidad de existencia de leishmaniasis visceral en Costa
Rica. Mem Inst Oswaldo Cruz. 1984;79(4):455-9.

19. Flores M, Martínez J, Gutiérrez R, Luna K, Serrano V, Ferro C, et al. Lutzomyia longipalpis (Diptera: Psychodidae) en un foco suburbano de leishmaniosis visceral en el Cañón del Chicamocha en Santander, Colombia. Biomedica. 2006;26(Supl. 1):109-20.

20. Salomón O, Rossi G, Spinelli G. Ecological Aspects of Phebotomine (Diptera, Psychodidae) in na Endemic Area of Tegumentary Leishmaniasis in the Northeastern
Argentina, 1993-1998. Mem Inst Oswaldo Cruz. 2002;97(2):163-8.

Correspondencia: Luis Eduardo Traviezo Valles

Dirección: Urbanización Tierra del Sol 2, Casa A-29, Cabudare, estado Lara, Venezuela. Código Postal 3023.

Teléfonos: (058) 0251 2591883, (058) 04145244736

Correo electrónico:luisetraviezo@hotmail.com



\section{ELISA de CAPTURA IgM DENGUE}

\section{TARIKI: disponible en el Perú}

Kit para la determinación de anticuerpos IgM anti Dengue, desarrollado y producido por el Instituto Nacional de Salud, Perú INS/MINSA 